\title{
Modified Location Model Estimation using Content Based Medical Image Retrieval
}

\author{
Sachin Kumar ${ }^{1} \&$ Krishna Prasad $K^{2}$ \\ ${ }^{1}$ CSE Dept., Hierank Business School, Noida, Affiliated to CCS University, Meerut, UP, India \\ ${ }^{2}$ College of Computer \& Information Science, Srinivas University, Mangalore, India. \\ E-mail: sachinks.78@,gmail.com
}

Area/Section : Computer Science.

Type of the Paper: Research Paper.

Type of Review: Peer Reviewed.

Indexed in: OpenAIRE.

DOI: http://doi.org/10.5281/zenodo.3411854.

Google Scholar Citation: IJMTS

How to Cite this Paper:

Kumar, Sachin. \& Krishna Prasad, K. (2019). Modified Location Model Estimation using Content Based Medical Image Retrieval. International Journal of Management, Technology, and Social Sciences (IJMTS), 4(2), 36-45. DOI: http://doi.org/10.5281/zenodo.3411854.

International Journal of Management, Technology, and Social Sciences (IJMTS)

A Refereed International Journal of Srinivas University, India.

IFSIJ Journal Impact Factor for $2018=4.764$

(C) With Authors.

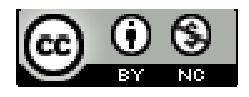

This work is licensed under a Creative Commons Attribution-Non Commercial 4.0 International License subject to proper citation to the publication source of the work.

Disclaimer: The scholarly papers as reviewed and published by the Srinivas Publications (S.P.), India are the views and opinions of their respective authors and are not the views or opinions of the SP. The SP disclaims of any harm or loss caused due to the published content to any party. 


\title{
Modified Location Model Estimation using Content Based Medical Image Retrieval
}

\author{
Sachin Kumar ${ }^{1}$ \& Krishna Prasad $K^{2}$ \\ ${ }^{1}$ CSE Dept., Hierank Business School, Noida, Affiliated to CCS University, Meerut, UP, India \\ ${ }^{2}$ College of Computer \& Information Science, Srinivas University, Mangalore, India. \\ E-mail: sachinks.78@gmail.com
}

\begin{abstract}
Image has become more and more difficult to process for human beings. Perfect results cannot be obtained through Content Based Medical Image Retrieval (CBMIR). The CBMIR was implemented to find order effectively retrieve the picture from an enormous database. Deep learning has taken Artificial Intelligence (AI) at an unprecedented rate through revolution and infiltration in the medical field. It has access to vast quantities of information computing energy of effective algorithms of Machine Learning (ML). It enables Artificial Neural Network (ANN) to attain outcomes nearly every Deep Learning (DL) problems. It helps ANN to achieve results everywhere. It is a difficult task to obtain medical images from an anatomically diff dataset. The goal of the research is to automate the medical image recovery scheme that incorporates subject and place probabilities to improve efficiency. It is suggested to integrate the different data or phrases into a DL location model. It is also measuring a fresh metric stance called weighted accuracy (wPrecision). The experiment will be conducted on two big medical image datasets revealing that the suggested technique outperforms current medical imaging technologies in terms of accuracy and mean accuracy. The CBMIR have about 8,000 pictures, the proposed technique will attain excellent precision (nearly 90 percent). The proposed scheme will attain greater precision for the top ten pictures ( 97.5 percent) as compared to the last CBMIR recovery technologies with 15,000 picture dataset. It will assist doctors with better accuracy in obtaining medical images.
\end{abstract}

Keywords: DL, Precision, Recall, CBMIR, AI, ANN, ML.

\section{PROBLEM :}

The CBMIR novel idea systems in non-medical fields have been also effective; there is a need to make successful CBMIR systems in medical domain. This research work aims to provide successful CBMIR system in medical domain by implementing and investigating various practices [1]. By analyzing and investigating these systems, a new scheme called Modified Location Model Estimation using Content Based Medical Image Retrieval has been proposed. The evaluation process, similar experimental setup has been implemented by implementing all the selected schemes on the same machine with identical test conditions [2]. Precision and recall were counted from the same reference database having 800 medical images, including local hospital and casImage database and IRMA database repositories from collected medical images from real-time medical images. Results of all the schemes were systematically analyzed, investigated [3]. It has been concluded from the results this work outperforms this scheme on content-based information retrieval under reduced processing time. It is inferred that the suggested system delivers $2 \%$ better accuracy rate and $5 \%$ better recall value at the same moment that processing requires $15 \%$ less time.

- To access distant pictures, we can use mobile image retrieval. 
- To access distant image LAN, WAN networks used.

- The retrieval time was reduced by incorporating the scheme in a hardware environment by designing suitable VLSI chips [4].

\section{INTRODUCTION :}

DL is a form of ML where a model predicts straight from pictures, text, or sound to conduct classification functions. Usually it is achieved using architecture of the neural network. "Deep" relates to the amount of network layers-the greater the network [5]. Technology has created strong techniques such as Topic Modeling to discover the hidden subjects in the data and collect relevant information from semantic sources. Topic Modeling is a method of recognizing the fundamental theme present in a document and obtaining patterns that are not seen in the records (pictures) to help make better decisions [6]. The present medical imaging recovery technologies rely on text-based recovery using the characteristics or patient information of DICOM. The recovery schemes based on text are unreliable, costly and susceptible to mistakes. CBMIR scheme allows skilled images getting from large database. Images are defined by their visual characteristics in CBMIR. The pictures contain these visual characteristics [7]. It is feasible to have low visual characteristics (colour, shape and texture). Topic Modeling Techniques Low-level visual characteristics for more significant subjects on a semantic basis. Topic Models are created from getting different words concepts representing a document as a word concept [8]. Topic Modeling decreases the low-dimensional topic of high-dimensional BoW. Visual words are a visual representation of function areas in pictures [9].

The word matrix of the document reflects the phrases LSA assumes the respective opinions A visual technique for estimating the topics is used by PLSA. In PLSA, from the collection papers, a topic distribution is learned. PLSA cannot design an invisible document, and PLSA's parameter count increases as the amount of documents increases. An amount of subjects is anticipated to be a specific value in PLSA and LDA techniques. Topic Modeling has recently been used in many medical image analysis and classification apps. Visual phrases generated using hair-like pictures from entire slide pictures of the medulloblastomatulers [10]. From these visual phrases, semantically more significant visual material is produced using PLSA to evaluate the pictures of histopathology. Every phrases, the elimination of insightful visual phrases and pictures is corrected correctly [11], proposed a scheme for medical image recovery with the combination of visual and textual information, the visual-textual word is formed. Zhang et al., Method based on pruned dictionary. To create a semantically more meaningful comparison of visual phrases, they calculate the meaning of the word and the complete meaning of the term [12]. Cao and Cao, mixed BoVW and PLSA technique for the classification of medical images. Zhang and others. new feature retrieval method suggested that in Visual Cases techniques are accessible. A major problem is that the image models will not appear in the verbal terms of the discriminating power's spatial information. Topic modeling was originally suggested for apps for text documents and subsequently adjusted to apps based on images [13]. A query was provided to the retrieval scheme for searching and retrieving pictures with comparable material in the internet retrieval method. The topic location characteristic is the query topic. The distinction between the topic location function and the expected topic pictures is then calculated using the measurement of Chi2 range $(\pi 2)$. Based on the measurement of computing range, the pictures are obtained and ranked.

\section{LITERATURE REVIEW :}

The findings of the semi-structured interviews disclosed that story components linked to environment, personality, plot, literary devices and topics were integrated in the majority of interpretations. The sequences of pictures were included in the components of the tale, suggesting that the modality model of the distinct pictures is very powerful. The technique of storytelling promotes the use of verbs and 
reactions to connotative levels. DL will make these images more accurate by using content based medical image [14]. Different pictures are closely curetted in DLs and several distinct metadata schemes are structured on distinct pictures.

Collecting pictures in a more fine-grained, structured database, DLs assist with distinct pictures to develop automatic methods for classification summary, visualization and content-based recovery. For the collection, it will educate many components of the collections, support, enrich and access various collections of pictures [15]. This research was created using a digital multiple picture to develop the inspection-repair process. This can improve FM procedures by enhancing efficiency and decreasing decision-making time and expenses. The system intended for automated pictures can be used in a smartphone, eliminates the time needed to manually input information pictures and increases communication and FM staff in the decision-making process for maintenance. This model helps to create excellent decisions [16]. Social networking website problems and hazards will also be placed forward in medical and every area, including defamation, privacy, data precision, and professional borders blurring. This model is also going to assist [17], the method of interpreting pictures was primarily linked to semance complement. DL's critical function in implementing pedagogy will remain more precise and consistent with the use of technology as well as related problems [18]. For the patient domain, the current domain-specific pre-trained word embedding would train it on a large database nearly five million patients to assess them. To conclude it, the writers will give a DL strategy for automatic patent classification on gated recurrent units based on the trained term embeddings [19]. Knowledge could be created by drawing inferences in a higher education institution for the assistance of personalized data in teaching and research. In the execution phase, the DL is practical limitations and true and accurate user concerns and efficiently maps the library's products and services [20].

\section{PROPOSED METHODS :}

In order to estimate words or phrases in subjects, here suggested location model [21-23]. The location function of a unique word in an picture I (Locationvi) is looking from the reference axis with range $(d)$. The place is characteristic of all $i$

Locationvi $=\{(\mathrm{d} v \mathrm{~m}, \theta \mathrm{v} \mathrm{m})$, for each $\mathrm{v} \in \mathrm{Vi}$

Locationi $=[\mathrm{v} \in \mathrm{ViLocationvi}$.

$\mathrm{C}$ is the number picture occurrences.

$\mathrm{Vi}$ is the collection of words \&

phrases in picture $\mathrm{i}$.

Database pictures are split in various queries i.e.into pictures. This method is mentioned as Al gorithm 1. Image of a picture based on the pictur e. Initially, pictures with theme pictures (TrainIt) If a visual term appears in $60 \%$ of images inTrainIt, it includes words and phrases [24]. Here suppose that distance (d) and angle (e.g.) of location characteristics are generally split into each cluster. Based on a maximum likelihood estimate, the parameters of each cluster's normal distribution ( $\mu \mathrm{d} v, \mu \mathrm{d} v, \mu \mathrm{ff} v$, $\mu \mathrm{ff} v, \mu \mathrm{ff} v$ ) are calculated.

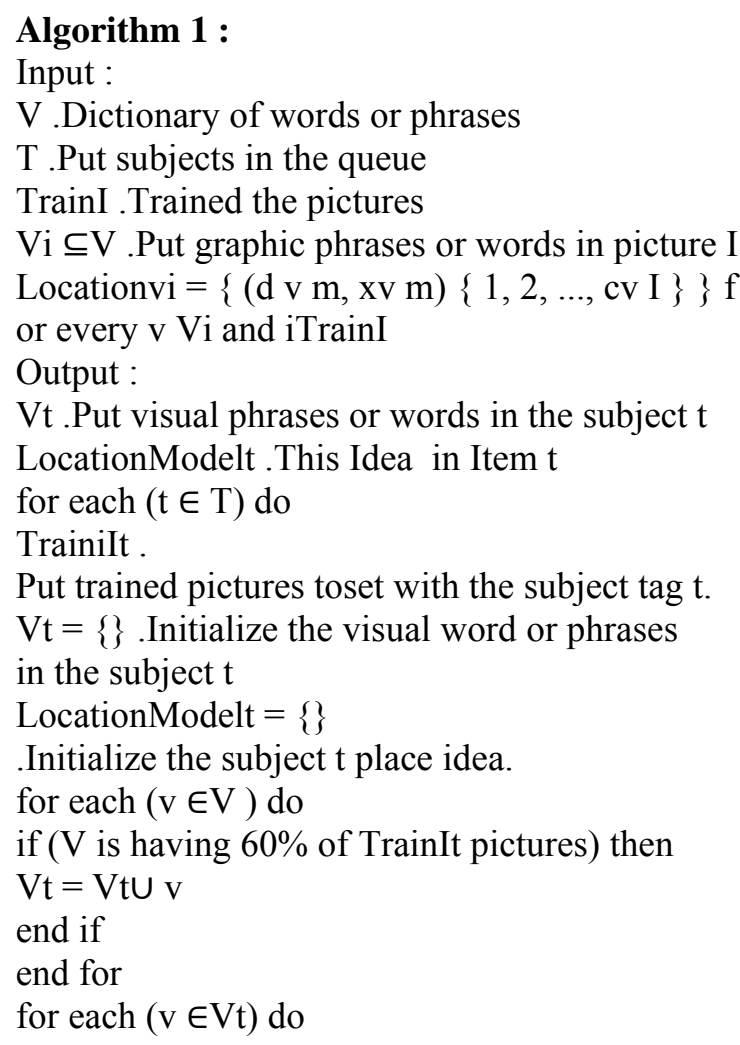




\section{LocationvTrainIt $=\{\}$ \\ for each ( $i \in$ TrainIt) do \\ $\mathrm{C}$ v TrainIt $[\mathrm{i}]=\mathrm{c}$ v $\mathrm{i}$}

.A range of $v$ events in TrainIt

LocationvTrainIt=LocationvTrainItULocationvi end for

$\mathrm{h}=\mid$ TrainIt $\mid$

$\mathrm{c} \mathrm{v} \mathrm{t}=$ FrequentCount $(\mathrm{C} v$ TrainIt, $\mathrm{h})$

In training pictures with subject label $t$, better $w$

ord or phrases with good frequency occurs at

good times.

Cluster LocationvTrainIt.

To find better estimate of probability,here it uses better parameters. [25]

[26]It is assumed that the distance between the $\mathrm{c}$ lusters is $N(\mu \mathrm{dv})$, and the angle between the clu sters is $N(\mu \mu v, \pi v)$

Location better word or phrases

model $\mathrm{v}$ into subject $\mathrm{t}$

LocationModelt $=$ LocationModelt $\mathrm{S}$

LocationModelv $\mathrm{t}$

end for

end for

ProcedureCountFrequent $(\mathrm{C} v$ TrainIt, h) Find th e most common $\mathrm{v}$ in TrainIt

for each $(i \in\{1,2,3, \ldots, h\})$

do

$\mathrm{c}=\mathrm{C}$ v TrainIt (i)

Histogram $(\mathrm{n})=\mathrm{n}$ rate in $\mathrm{N} v$ TrainIt. Calculate $\mathrm{y}$ our histo

gram

end for

Histogram(n) $\mathrm{z}=\operatorname{argmax} \mathrm{n}$. The most common $\mathrm{i}$ ncidence of $v$ in TrainIt

return $\mathrm{z}$

end procedure

Algorithm2:

Estimate Place likelihood of picture I in idea Input :

$\mathrm{V}$.Dictionary of words or phrases

$\mathrm{Vi} \subseteq \mathrm{V}$..Set of graphic phrases in picture I

Locationvi $=\{(\mathrm{d} v \mathrm{~m}, \mathrm{xv} \mathrm{m})\{1,2, \ldots, \mathrm{cv} \mathrm{I}\}\} \mathrm{f}$ or every single $\mathrm{v}=\mathrm{V}$

Better words v location characteristics in pictur.

T . Put subjects in idea

$\mathrm{Vt} \subseteq \mathrm{V}$.Put of better words or phrases

in present in Item $t$

LocationModelv $\mathrm{t}=\{(\mathrm{N}(\mu \mathrm{d} \mathrm{v} \mathrm{n}), \mathrm{N}(\mu \mu \mathrm{v} v \mathrm{n})$ $, \mathrm{n}\{1,2, \ldots, \mathrm{cv} \mathrm{t}\}\}$ for every $\mathrm{v} \mathrm{V}$
Output :

$\mathrm{p}(\mathrm{lt} \mid \mathrm{v}, \mathrm{i})$. Location likelihood of better words or phrases in subject for a specified picture.

for each $(\mathrm{t} \in \mathrm{T})$ do

for each $(v \in V)$ do

$\mathrm{p}(\mathrm{lt} \mid \mathrm{v}, \mathrm{i})=0$. Initialize this chance

if $(\mathrm{v} \in \mathrm{Vt} \cap \mathrm{Vi})$ then

$\mathrm{P}(\mathrm{lt}, \mathrm{i})=$ ProbabilityLocation(ProbabilityLocation

Modelv $\mathrm{t}$ )

end if

end for

For every words or phrases in the dictionary, it cosidered uniform $\mathrm{P}(\mathrm{v})=1$

end for

procedureLocationProbability(Locationvi

,LocationModelv t )

MatchCount $=0$.Initialise $M$ atchCount

Locationvi $=\{(\mathrm{d} v \mathrm{~m}, \theta \mathrm{v} \mathrm{m}) \mid \mathrm{m}$.

Better word or

phrases characteristics in picture I with

its occurrences of picture.

Location characteristics of the better words or phrases in the subject occures in the subject.

TotalCount $=\mathrm{c} \mathrm{v} \mathrm{i}+\mathrm{c} \mathrm{v} \mathrm{t}$.

Maximum number of occursin picture and subje ct

$\mathrm{E}[1: \mathrm{c} v \mathrm{i}][1: \mathrm{c} v \mathrm{t}]=0$.

Verify thisdifference inbetween mthLocationvi a nd nth position.

if $(\delta \mathrm{d} v(\mathrm{~m}, \mathrm{n}) \leq 2 \sigma \mathrm{d} v \mathrm{n})$ and $(\delta \theta \mathrm{v}(\mathrm{m}, \mathrm{n}) \leq 2 \sigma$

$\theta \vee \mathrm{n})$ then

$\mathrm{E}[\mathrm{m}, \mathrm{n}]=1$ Member. $\mathrm{E}[\mathrm{m}, \mathrm{n}]=1$

end if

Build a G(L, R, E) bipartate chart.

Find the Emax of $\mathrm{G}$ maximum

MatchCount $=2 * \mid$ Emax $\mid$

There are two matching places in each game

$\mathrm{p}(\mathrm{lt} \mid \mathrm{v}, \mathrm{i})=\mathrm{M}$ atchCount $/ \mathrm{T}$ otalCount

return $\mathrm{p}(\mathrm{lt} \mid \mathrm{v}, \mathrm{i})$

end procedure

\section{WEIGHTED PRECISION :}

Medical image recovery system recovery performance The doctors are the only ones to get rid of the system. Precision @ Q or ' Precision at $\mathrm{Q}^{\prime}$ reflects the first $\mathrm{Q}$ pictures that have been obtained [27]. The standard Precision (a) $\mathrm{Q}$ cannot capture the rank of the pictures that have been obtained. In this job, we will use two 
big anatomically distinct database which evaluate for retrieval efficiency for proposed scheme [28]. The proposed technique will have two parameters, the K-means cluster center $(\mathrm{K})$, which will determine the amount of graphic phrases in the dictionary and the amount of subjects that will determine the place feature vector length subject [29]. We begin each other from threshold distance (euclidean) with cluster centers to avoid, thus having the better words or phrases. It is based on the database and the number of subjects in each information set is chosen [30]. It runs on Python or Matlab software is used to execute the suggested technique. The Precision-Recall curve will graphically depict the accuracy and recall values by plotting accuracy in the order positioned at each recall level.

Precision $=$ Number of pictures collected $/$ Total number of pictures collected.

Recall $=$ Number of appropriate pictures / Total number of appropriate pictures

\section{EXPERIMENT 1 :}

The medical pictures have found in more than 24 different areas to form the database from publicly accessible medical pictures [31]. The Cancer Imaging Archive will collect twenty-two anatomical areas. The dataset comprises of 300 pictures in each anatomical region, 70 percent pictures for randomly chosen practice from each anatomical region, and the remaining 30 percent pictures for use [32]. The Multimodal dataset will consist of 7200 pictures, which will be split into 5040 training set pictures and 2160 test set query pictures. The amount of cluster centers, or the dictionary's better words or phrases, would be more than 60 using a minimum Euclidean distance 600 between clusters [33]. The amount in topics for database will be allocated as 30 depending in between the database's various anatomical areas. Here, using Deep Learning, these techniques will assess total efficiency with suggested scheme using CBMIR methods. In the latest Deep Convolution Neural Network (DeepCNN), we will compare [34]. The achievement of DeepCNN was evaluated with a comparable database where more than 23 different areas were the same as the database.
We will compare DeepCNN's output with the technique of the proposition. We compared to the expected topic pictures. Similarly, we will use the likelihood of subject in GuidedLDA. 70 percent of pictures from each body organ will be chosen randomly to generate the model in each run and the remaining 30 percent will be used as a request [35]. The execution of the pictures and query picture of the forecast of the retrieval item label with enhancement performance are better. The strategy also decreases the search demands for the appropriate pictures in the dataset as a further benefit. Various topic modeling and dictionary encoding methods will also be used to evaluate the efficiency of the proposed scheme using the same dataset. The efficiency of the label will be evaluated in BoVW and PLSA technique, BoVW would be built using descriptors from SIFT, and the topic might use PLSA as a vector [36].

\section{EXPERIMENT 2 :}

It will be gray-scale X-ray pictures of separate different areas on distinct modalities and orientations. The technique of performance evaluation for 57 anatomical areas from 14,410 pictures will be proposed using 2005 coding scheme based on IRMA pictures [37]. The amount of clusters will be nearly 80 by the distance between clusters of Threshold 600 . The database subjects will be allocated as 57 depending on the data set's distinct body components. Initially, the expected theme label's function database in the pictures that contain the pictures. To assess the efficiency of the suggested technique, one hundred pictures will be randomly chosen from the dataset. Sparse Dictionary Learning technique will use the mean and variance of pixel intensities, then comparison outcomes of the proposed scheme with the CBMIR technique using different database will be provided. The picture recovery system efficiency will need to be used. Using Precision@Q, image ranking in the return list will typically be evaluated using accuracy at a specific cut-off Q. But this analyze the ranking order of the returned pictures, this will obviously be more effective to guide the 
retrieval of the suggested technique than the Guided LDA [38].

\section{OVERCOME THE PROBLEM :}

Modified Location Model Estimation using Content Based Medical Image Retrieval has been implemented for improvement. To show the consistency of the project, throughout the execution process, the utmost care has been taken to ensure that the same type of experimental setup, similar comparison technique, and reference database used [39]. To compare the throughput of the proposed scheme, similar experimental setup both schemes implemented by on the same machine with identical test conditions. Precision and recall were calculated from the same reference database having 800 or more medical images that include real-time medical images collected from local hospital and medical images from collected CasImage database and IRMA database repositories. It results from the conclusion that the proposed scheme is outperforms the content on the current scheme based information retrieval under less processing time. It is inferred that the proposed scheme deliver $2 \%$ better precision level and 5\% better recall value at the same time it takes $15 \%$ lesser time for processing [40].

- The CBMIR provides numerous unique appli cations.

- It is also providing distinctive picture characteristics.

- To provide a smart medical imaging system $\mathrm{t}$ o support medical diagnosis

- By making measurement using conventional measurement factors namely precision and recall, the system will provide the image retrieval process. That is the image of the database

- The system offers the picture recovery schem e with scalability and velocity efficiency.

- Precision and recall were calculated using the same reference database with 800 or more m edical images, including realtime medical im ages, gathered from local hospital and medic al images gathered from CasImage database and repositories from IRMA database.

\section{CONCLUSION :}

The increasing proliferation of internet in all human activities and the advancement of imaging technologies made drastic changes. There are huge image repositories in various Universities, Government agencies, hospitals, forensic departments, financial institutions etc. In the Knowledge era, information and data becomes open source. It allows users to use the image data from the repositories for their research, analysis, verification, authentication etc. But, getting the database picture recovery systems to enable the user to define their requirement or by using a comparable query picture is a challenging job. In the context of images, the annotation-based retrieval systems are not effective because the retrieval process is trying to match. The imprecise text is also a challenging job [41]. Medical image retrieval based on content is based on picture characteristics The picture can be discovered in the Occlusion and Rotation picture. For a subject-location feature vector, anatomically distinct big multimodal medical image datasets are obtained from pictures. The topic-location function is acquired through the probabilities of topic and location. The suggested place model will be depicted in the pictures of each topic as a standard distribution of pictures. Here we will calculate the probability location of a picture based on location closeness [42]. Again, we will predict the request from the vector of the subject location function, which decreases the search time by avoiding the complete pictures in the entire dataset. The practical results clearly emphasize the inclusion of retrieval systems in the information related to the subject-of - theart. In this we will concentrate on medical image retrieval and anomaly size. Such systems can automate the process of diagnosing and forecasting lethal illnesses step-by-step. In addition, such a recovery scheme will also allow physicians to conduct a comparative assessment. We will generate the dictionary using the SIFT descriptors ' K-Means clustering, and every cluster core includes a better words or phrases for the dictionary. We will use a straightforward point of perspective (SCS) algorithm using the seed point initialization technique to avoid the 
random initialization generation of graphic phrases with comparable structure. Instead of continually initializing the seed points in SCS, It will allow users for regulate this distinct cluster centers with distance (using threshold distance) and set sequence order information for helpgenerating fixed cluster centers. In the technique of initialization of seed points, Instead of the amount of clusters the customer must indicate a limit distance. The steps used in the initialization of seeds are given below;

1. Collect descriptors for SIFT

2. Initialize the list of seed points as vacant.

3. Initialize the collection's first SIFT discriptor

4. If the Euclidean distance is higher than the Threshold range

\section{(a) Add descriptor}

Else

(b) Rotate descriptor

5. Repeat its step4 for all collection SIFT descriptors.

6. Perform KMeans clustering in the set of SIFT descriptors by initializing the seed points using the list of seed points. Using 1,000 pictures from the dataset will be performed to discover the ideal limit distance from an experiment. One hundred pictures from ten anatomical areas will be randomly chosen, and the clusters start with random starting threshold distance (650, 700, 750) using SCS. Analysis of the clusters created by the Calinski-Harabasz Index (CHI) will select the optimum limit distance. By improving the CHIK percentage, information partitioning could be enhanced. It is initialized in information to be clustered after initializing the threshold distance. If there are comparable few clusters to form the information or patches obtained from pictures, otherwise more clusters will be developed [43].

\section{FUTURE SCOPE :}

For future studies, content-based medical image recovery systems are prominently accessible. This study work enhances the knowledge of various methods for extraction of features and similarity measurements that help to retrieve medical pictures through their contributions. The inclusion of different methods described in this study job poses a number of difficulties for further studies, such as validating the practicality of the suggested model in actual surroundings: using a realistic medical environment by considering various priorities for digital medical images. Incorporate more advanced methods for the recovery of medical image [44].

\section{REFERENCES :}

[1] Mustra, M., Delac, K., \& Grgic, M.(2008). Overview of the DICOM standard. In 2008 50th International Symposium ELMAR (Vol. 1, pp. 39-44). IEEE.

[2] Akgül, C. B., Rubin, D. L., Napel, S., Beaulieu, C. F., Greenspan, H., \& Acar, B. (2011). Content-based image retrieval in radiology: current status and future directions. Journal of digital imaging, 24(2), 208-222.

[3] Kumar, A., Kim, J., Cai, W., Fulham, M., \& Feng, D. (2013). Content-based medical image retrieval: a survey of applications to multidimensional and multimodality data. Journal of digital imaging, 26(6), 10251039.

[4] Csurka, G., Dance, C., Fan, L., Willamowski, J., \& Bray, C. (2004). Visual categorization with bags of keypoints. In Workshop on statistical learning in computer vision, ECCV (Vol. 1, No. 1-22, pp. 1-2).

[5] Dumais, S. T. (2004). Latent semantic analysis. Annual review of information science and technology, 38(1), 188-230.

[6] Hofmann, T. (2000). Learning the similarity of documents: An information-geometric approach to document retrieval and categorization. In Advances in neural information processing systems (pp. 914-920).

[7] Blei, D. M., Ng, A. Y., \& Jordan, M. I. (2003). Latent dirichlet allocation. Journal of machine Learning research, 3(Jan), 993-1022.

[8] Castellani, U., Perina, A., Murino, V., Bellani, M., Rambaldelli, G., Tansella, M., \& Brambilla, P. (2010). Brain morphometry by probabilistic latent semantic analysis. In International Conference on Medical Image 
Computing and Computer-Assisted Intervention (pp. 177-184). Springer, Berlin, Heidelberg.

[9] Foncubierta-Rodríguez, A., García Seco de Herrera, A., \& Müller, H. (2013). Medical image retrieval using bag of meaningful visual words: unsupervised visual vocabulary pruning with PLSA. In Proceedings of the 1st ACM international workshop on Multimedia indexing and information retrieval for healthcare (pp. 7582). ACM.

[10] Foncubierta-Rodríguez, A., García Seco de Herrera, A., \& Müller, H. (2013). Medical image retrieval using bag of meaningful visual words: unsupervised visual vocabulary pruning with PLSA. In Proceedings of the 1st ACM international workshop on Multimedia indexing and information retrieval for healthcare (pp. 7582). ACM.

[11] Cao, Y., Steffey, S., He, J., Xiao, D., Tao, C., Chen, P., \& Müller, H. (2014). Medical image retrieval: a multimodal approach. Cancer informatics, 13, CIN-S14053.

[12] Zhang, F., Song, Y., Cai, W., Hauptmann, A. G., Liu, S., Pujol, S., ... \& Chen, M. (2016). Dictionary pruning with visual word significance for medical image retrieval. Neurocomputing, 177, 75-88. .

[13] Cao, C. H., \& Cao, H. L. (2016). The research on medical image classification algorithm based on PLSA-BOW model. Technology and Health Care,24(s2), S665-S674.

[14] Zhang, F., Song, Y., Cai, W., Liu, S., Liu, S., Pujol, S., ... \& Alzheimer's Disease Neuroimaging Initiative. (2015). Pairwise latent semantic association for similarity computation in medical imaging. IEEE transactions on Biomedical Engineering, 63(5), 1058-1069.

[15] Y. Ma, Z. Jiang, H. Zhang, F. Xie, Y. Zheng, H. Shi, Y. Zhao (2017), Breast histopathological image retrieval based on latent dirichlet allocation, IEEE journal of biomedical and health informatics, 21 (4), 1114-1123.

[16] Jagarlamudi, J., Daumé III, H., \& Udupa, R. (2012). Incorporating lexical priors into topic models. In Proceedings of the 13th Conference of the European Chapter of the Association for Computational Linguistics (pp. 204-213). Association for Computational Linguistics.

[17] Deza, M. M., Deza, E. (2009). Encyclopedia of distances. 2009.

[18] Lowe, D. G. (2004). Distinctive image features from scale-invariant keypoints. International journal of computer vision, 60(2), 91-110.

[19] Srinivas, M., Naidu, R. R., Sastry, C. S., \& Mohan, C. K. (2015). Content based medical image retrieval using dictionary learning. Neurocomputing, 168, 880-895.

[20] Camlica, Z., Tizhoosh, H. R., \& Khalvati, F. (2015). Autoencoding the retrieval relevance of medical images. In 2015 International Conference on Image Processing Theory, Tools and Applications (IPTA) (pp. 550-555). IEEE.

[21] Welter, P., Fischer, B., Günther, R. W., \& Deserno, T. M. (2012). Generic integration of content-based image retrieval in computer-aided diagnosis. Computer methods and programs in biomedicine, 108(2), 589-599.

[22] Tou, J. T., \& Gonzalez, R. C. (1974). Pattern recognition principles.

[23] Caliński, T., \& Harabasz, J. (1974). A dendrite method for cluster analysis. Communications in Statistics-theory and Methods, 3(1), 1-27.

[24] Manning, C., Raghavan, P., Schuutze, H. (2008). Introduction to Information Retrieval, An Introduction to Information Retrieval, Cambridge University Press, 2008.

[25] Clark, K., B. Vendt, K. Smith, J. Freymann, J. Kirby, P. Koppel, S. Moore, S. Phillips, D. Maffitt, M. Pringle (2013). The cancer imaging archive (tcia): maintaining and operating a public information repository, Journal of digital imaging, 26 (6), 1045-1057.

[26] Decenci`ere, E., X. Zhang, G. Cazuguel, B. Lay, B. Cochener, C. Trone, P. Gain, R. Ordonez, P. Massin, A. Erginay. (2014). Feedback on a publicly distributed image database: the messidor database, Image Analysis 
\& Stereology, 33 (3), 231-234.

[27] Qayyum, A., Anwar, S. M., Awais, M., \& Majid, M. (2017). Medical image retrieval using deep convolutional neural network. Neurocomputing, 266, 8-20.

[28] Sánchez, J., Perronnin, F., Mensink, T., \& Verbeek, J. (2013). Image classification with the fisher vector: Theory and practice. International journal of computer vision, 105(3), 222-245.

[29] Spyromitros-Xioufis, E., Papadopoulos, S., Kompatsiaris, I. Y., Tsoumakas, G., \& Vlahavas, I. (2014). A comprehensive study over VLAD and product quantization in largescale image retrieval. IEEE Transactions on Multimedia, 16(6), 1713-1728.

[30] Shamna, P., Govindan, V. K., \& Nazeer, K. A. (2019). Content based medical image retrieval using topic and location model. Journal of biomedical informatics, 91, 103112.

[31] Hogg, R. V., \& Ledolter, J. (1987). Engineering statistics. Macmillan Pub Co.

[32] Hochberg, J., \& TAMHANE, A. C. (1987). Multiple comparison procedures (No. 519.535 H655m Ej. 1). John Wiley \& Sons.

[33] Lehmann, T. M., Schubert, H., Keysers, D., Kohnen, M., \& Wein, B. B. (2003). The IRMA code for unique classification of medical images. In Medical Imaging 2003: PACS and Integrated Medical Information Systems: Design and Evaluation (Vol. 5033, pp. 440-451). International Society for Optics and Photonics.

[34] Shamna, P., Govindan, V. K., \& Nazeer, K. A. (2019). Content based medical image retrieval using topic and location model. Journal of biomedical informatics, 91, 103112.

[35] Rafferty, P., \& Albinfalah, F. (2014). A tale of two images: the quest to create a story-based image indexing system. Journal of Documentation, 70(4), 605-621.

[36] Qu, J., \& Chen, J. (2019). An investigation of benchmark image collections: how different from digital libraries?. The Electronic Library.

[37] Zhan, J., Ge, X. J., Huang, S., Zhao, L.,
Wong, J. K. W., \& He, S. X. (2019). Improvement of the inspection-repair process with building information modelling and image classification. Facilities, 37(7/8), 395-414.

[38] Lebow, D. G., Lick, D. W., Hartman, H. J., Dalglish, C., \& Grundmann, O. (2011). Social annotation to enhance learning and assessment in higher education. In Educating Educators with Social Media (pp. 261-278). Emerald Group Publishing Limited.

[39] Lim, W. M. (2016). Social media in medical and health care: opportunities and challenges. Marketing intelligence \& planning, 34(7), 964-976.

[40] Li, X., Wu, Y., Wang, X., Qian, T., \& Hong, L. (2019). Towards a semantics representation framework for narrative images. The Electronic Library.

[41] Bower, M. (2017). Design of technologyenhanced learning: integrating research and practice. Emerald Publishing Limited.

[42] Davies, R. (1989). The creation of new knowledge by information retrieval and classification. Journal of documentation, 45(4), 273-301.

[43] Davies, R. (1989). The creation of new knowledge by information retrieval and classification. Journal of documentation, 45(4), 273-301.

[44] Sirisha, B. S., Jeevan, V. K. J., Raja Kumar, R. V., \& Goswami, A. (2009). A personalised information support system for searching portals and eresources. Program, 43(1), 77-93. 